References:

[1] Voo KS, et al. Proc Natl Acad Sci U S A. 2009;106:4793.

[2] Beriou G et al. Blood. 2009;113:4240.

[3] Wang T, et al. Ann Rheum Dis. 2015;74:1293.

[4] Komatsu N, et al. Nat Med. 2014;20:62.

[5] Yang BH, et al. Mucosal Immunol. 2016;9:444.

Disclosure of Interest: None declared

DOI: 10.1136/annrheumdis-2017-eular.4088

\section{FRI0063 RESVERATROL ATTENUATES SYNOVIAL HYPERPLASIA IN AN ACUTE ANTIGEN-INDUCED ARTHRITIS MODEL BY AUGMENTING AUTOPHAGY AND DECREASING ANGIOGENESIS}

M. Almonte-Becerril ${ }^{1}$, J.A. Fernandez-Rodriguez ${ }^{1}$, J.A. Fernandez-Rodriguez ${ }^{1}$, O. Ramil-Gomez ${ }^{1}$, R.R. Riveiro-Naveira ${ }^{1}$, L. Hermida-Carballo ${ }^{1}$, A. Concha ${ }^{2}$, A. Vela-Anero ${ }^{3}$, S. Viñas ${ }^{1}$, F.J. Blanco ${ }^{4}$, M.J. Lopez-Armada ${ }^{1} .{ }^{1}$ Aging and Inflammatory Research Lab; ${ }^{2}$ Servicio de Patología; ${ }^{3}$ Terapia Celular y Medicina Regenerativa; ${ }^{4}$ Laboratorio de Investigacion Osteoarticular y Envejecimiento, SERGAS, A Coruña, Spain

Background: Previously, we have demonstrated that dietary supplementation with resveratrol lowers synovial hyperplasia, inflammatory markers and oxidative damage in an acute antigen-induced arthritis (AIA) model.

Objectives: In this work, we investigated whether resveratrol can also regulate this abnormal proliferation of synovial tissue in an acute AIA model by inducing cell death pathways and by modifying the angiogenesis in the synovial membrane. Methods: Animals were randomly divided into 3 groups: control, AIA, and resveratrol-treated AIA group. Resveratrol $(12,5 \mathrm{mg} / \mathrm{kg} /$ day $)$ was given orally 8 weeks before AIA induction until sacrifice day ( $48 \mathrm{~h}$ after intra-articular injection). Control and AIA animals were administered $100 \mu \mathrm{l}$ of water. Resveratrol effects on autophagy and apoptosis were evaluated by LC3 and active caspase-3 expression (confocal and immunohistochemistry, respectively). Angiopoietin 1 (Ang-1), vascular endothelial growth factor (VEGF), and the nuclear factor NFkappa-B p65 subunit (p65) were also determined by immunohistochemistry and cartilage degradation with Safranin-O.

Results: Resveratrol significantly reduced the histological score of synovial tissue. Results showed a significant higher expression of LC3 signals in the AIA synovial membranes, compared with control samples, in which the presence of vesicles was easily observed. Interestingly, the synovial tissues from the resveratrol group showed a significantly $(p \leq 0.001)$ higher signal for LC3, compared with the AIA samples. Active caspase-3 expression was up-regulated at the same level in the synovial membranes of AIA group than in resveratrol-treated AIA group; however, in resveratrol-treated AIA group active caspase- 3 signal was mainly located in the inflammatory cells. Resveratrol consumption significantly attenuated Ang-1 signal, whereas expression of VEGF showed a non significant reduction. Resveratrol administration also mitigated, even not significantly, p65 expression that was significantly higher in the AIA animals than those from the control animals. In addition, resveratrol decreased articular cartilage degradation.

Conclusions: These data suggest that resveratrol is able to modulate synovial hyperplasia by increasing autophagic cell death and limiting angiogenic response in an acute AIA model, which could also modulate the inflammatory and destructive processes for rheumatoid arthritis.

Acknowledgements: Instituto de Salud Carlos III-Ministerio de Economía y Competitividad N Expediente PI12/02771. Unión Europea-Fondo Europeo de Desarrollo Regional (FEDER) "Una manera de hacer Europa"

Disclosure of Interest: None declared

DOI: 10.1136/annrheumdis-2017-eular.6270

\section{FRI0064 CHEMOKINE RECEPTOR 6 MODULATES ARTHRITIS IN A T CELL DEPENDENT MANNER}

M. Bonelli, A. Puchner, L. Goeschl, S. Hayer, B. Niederreiter, J.S. Smolen, C. Scheinecker, S. Blueml. Devision of Rheumatology, Medical University of Vienna, Vienna, Austria

Background: Rheumatoid arthritis (RA) is an inflammatory autoimmune disease, characterized by synovial infiltration of various cells. Chemokines are involved in the recruitment of different cell types into the synovial membrane. Accumulation of CCR6 expressing mononuclear cells can be found in joints of RA patients. CCR6 expression has also been reported on $\mathrm{CD} 4^{+} \mathrm{T}$ cells, in particular regulatory as well as Th17 cells. In addition, a subset of regulatory $\mathrm{T}$ cells, namely CD25-Foxp3 ${ }^{+}$T cells, can upregulate CCR6 and RANKL and thereby can promote osteoclastogenesis.

Objectives: In this study, we investigated the role of CCR6 in the pathogenesis of arthritis using different arthritis models.

Methods: Clinical as well as histological signs of arthritis were investigated in the collagen-induced arthritis (CIA), K/BxN serum transfer arthritis and in the human tumor necrosis factor (hTNFtg) arthritis model, comparing wt and CCR6-/- mice. We analyzed the phenotype of lymph node cells by flow cytometry and cytokine concentrations in serum. Anti-collagen antibodies and cytokines were measured by enzyme-linked immunosorbent assay.

Results: The K/BxN serum transfer arthritis and hTNFtg arthritis model are known to be T cell independent. Since CCR6 is an important component of the innate immune system we compared the development of arthritis in both models. We did not detect any significant differences in clinical signs of inflammation or histological severity of arthritis between wt and CCR6-/- mice. In addition, bone volume was similar between $w t$ and CCR6-/- mice. To investigate the role of CCR6 as part of the adaptive immune system in the development of arthritis we induced CIA in wt and CCR6-/- mice, which is known to be T cell dependent. CCR6-/- mice were almost completely protected from CIA. Indeed, analyses of T cell subsets by flow cytometry revealed a significant reduction of $\mathrm{CD}^{-} 5^{-} \mathrm{Foxp}^{+} \mathrm{T}$ cells.

Conclusions: CCR6 is necessary for the generation of pathogenic $\mathrm{CD}^{2} 5^{-} \mathrm{Foxp}^{+}$ T cells in CIA, suggesting an important function of CCR6 on T cells in the development of autoimmune arthritis.

Disclosure of Interest: None declared

DOI: 10.1136/annrheumdis-2017-eular.5107

\section{FRI0065 HELMINTHES BASED NOVEL COMPOUND, TUFTSIN-PHOSPHORYLCHOLINE (TPC) AMELIORATES ESTABLISHED MURINE ARTHRITIS}

T. Bashi ${ }^{1}$, J. Lachnish ${ }^{1}$, M. Fridkin ${ }^{2}$, Y. Shoenfeld ${ }^{3}$, M. Blank ${ }^{1} .{ }^{1}$ Zabludowicz Center for Autoimmune Diseases, Sheba Medical Center, Ramat Gan; ${ }^{2}$ Organic Chemistry, Weizmann, Rehovot; ${ }^{3}$ Zabludowicz Center for Autoimmune Diseases, Sheba.health.gov.il, Ramat Gan, Israel

Background: In areas where helminthes infections are common, autoimmune diseases are rare. Treatment with helminthes or their ova, improved clinical findings of several autoimmune diseases. Based on the helminthes biological activity, a novel chimeric be-specific molecule was synthetized of tuftsin-phosphoryl-choline (PC)-TPC.

Objectives: To study TPC treatment in established collagen induced arthritis (CIA) mice, and the mechanism of activity.

Methods: Arthritis was induced in DBA male mice by immunization with collagen emulsified in TB-mycobacteria at the tail base. Boost was given 3 weeks later. Treatment with TPC started when the clinical score was 2. Cytokines were measure in culture-fluid of splenocytes in-vitro. T regulatory cells and B regulatory cells were measured by FACS. TPC effect on TLR4 expression was studied using HEKTM-mTLR4 cells system and its inhibitor. M1 shift to M2 was performed in RAW macrophages differentiated to M1 by PMA followed by LPS. TPC was added and IL-6, TNFalpha, IL-10 were tested by ELISA.

Results: Starting TPC treatment of CIA mice after disease establishment, had a significant lower arthritis score in comparison with control vehicle subjected mice (i.e. TPC- $6.8 \pm 0.8$ vs vehicle- $13.8 \pm 0.45 ; p<0.0001$ ). Joints staining revealed normal joint structure in TPC treated mice, whereas, control mice treated with PBS, PC or tuftsin had severe inflamed joints. Likewise, TPC enhanced antiinflammatory response by enhanced IL-10 secretion, reduced pro-inflammatory cytokines secretion (IL-1 $1, \mathrm{IL}-17, \mathrm{IL}-6$, and TNF- $\alpha)(\mathrm{p}<0.001)$. Furthermore, TPC induced expansion of splenic CD4+CD25+FOXP3+ T regulatory cells (Tregs) and IL-10+CD5+CD1d+B regulatory cells (Bregs). The mechanism underling the TPC related immunomodulatory activity was attributed to its bi-specific activity: a) Shift of Raw cells macrophages from pro-inflammatory macrophages M1 to anti-inflammatory M2 secreting anti- IL-10 $(p<0.001)$, through the tuftsin part of TPC. b) TPC inhibited significantly TLR4 expression by HEKTM-mTLR4 cells $(p<0.02)$ via the phosphorylcholine end. Our data indicated that TPC significantly ameliorated established CIA by anti-inflammatory immunomodulatory activity.

Conclusions: Our data may lead to a novel bi-specific self small molecule for therapy of patients with advanced RA.

Disclosure of Interest: None declared

DOI: 10.1136/annrheumdis-2017-eular.3065

\section{FRI0066 GASTRIN-RELEASING PEPTIDE AND ITS RECEPTOR INCREASE ARTHRITIS FIBROBLAST-LIKE SYNOVIOCYTE INVASIVENESS OVER THE PI3K/AKT PATHWAY}

M. Farinon ${ }^{1,2}$, V.S. Clarimundo ${ }^{1}$, R.T. Pedó ${ }^{1,2}$, V.O.N. Teixeira ${ }^{1}$, C. Nör ${ }^{3}$, P.S. Gulko ${ }^{4}$, R.M. Xavier ${ }^{1,2}$, P.G. Oliveira ${ }^{1,2}{ }^{1}$ Hospital de Clínicas de Porto Alegre; ${ }^{2}$ Universidade Federal do Rio Grande do Sul, Porto Alegre, Rio Grande do Sul, Brazil; ${ }^{3}$ Peter Gilgan Centre for Research and Learning, Toronto, Ontario, Canada; ${ }^{4}$ Icahn School of Medicine at Mount Sinai, New York, United States

Background: Rheumatoid arthritis (RA) is an autoimmune disease where the chronic inflammation and subsequent cartilage and bone erosion lead to joint destruction. The fibroblast-like synoviocytes (FLS) have a central role in disease pathogenesis and in vitro FLS invasiveness correlates with articular damage in RA patients. Gastrin-releasing peptide (GRP) plays an important role in the immune and inflammatory response. GRP is found in synovial fluid of RA patients and its receptor (GRPR) is found in synovial membrane of murine arthritis. RC-3095 in an antagonist of GRPR.

Objectives: To evaluate the role of GRP and GRPR on invasive behavior of mice FLS and to evaluate the GRP-induced signaling on PI3K/AKT pathway.

Methods: FLS were isolated from joints of DBA/1J mice with collagen-induced arthritis. Expression of GRPR in FLS was investigated by immunocytochemistry and western blot (WB). Proliferation of FLS treated with GRP $(0.1 \mu \mathrm{M}-10 \mu \mathrm{M})$ 